\title{
Preliminary Results from the Caltech Core-Collapse Project (CCCP)
}

\author{
Avishay Gal-Yam*, ${ }^{* \dagger}$, S. B. Cenko*, D. B. Fox**, D. C. Leonard ${ }^{\ddagger}$, D.-S. \\ Moon*, D. J. Sand ${ }^{\S}$ and A. M. Soderberg* \\ *Division of Physics, Mathematics and Astronomy, California Institute of Technology \\ ${ }^{\dagger}$ Hubble Fellow \\ ${ }^{* *}$ Department of Astronomy and Astrophysics, Pennsylvania State University \\ ${ }^{\ddagger}$ Department of Astronomy, San Diego State University \\ ${ }^{\S}$ Steward Observatory, University of Arizona
}

\begin{abstract}
.
We present preliminary results from the Caltech Core-Collapse Project (CCCP), a large observational program focused on the study of core-collapse SNe. Uniform, high-quality NIR and optical photometry and multi-epoch optical spectroscopy have been obtained using the 200" Hale and robotic 60" telescopes at Palomar, for a sample of 50 nearby core-collapse SNe. The combination of both well-sampled optical light curves and multi-epoch spectroscopy will enable spectroscopically and photometrically based subtype definitions to be disentangled from each other. Multi-epoch spectroscopy is crucial to identify transition events that evolve among subtypes with time. The CCCP SN sample includes every core-collapse SN discovered between July 2004 and September 2005 that was visible from Palomar, found shortly ( $<30$ days) after explosion (based on available preexplosion photometry), and closer than $\sim 120 \mathrm{Mpc}$. This complete sample allows, for the first time, a study of core-collapse $\mathrm{SNe}$ as a population, rather than as individual events. Here, we present the full CCCP SN sample and show exemplary data collected. We analyze available data for the first $\sim 1 / 3$ of the sample and determine the subtypes of $13 \mathrm{SNe}$ II based on both light curve shapes and spectroscopy. We discuss the relative SN II subtype fractions in the context of associating SN subtypes with specific progenitor stars.
\end{abstract}

Keywords: Supernovae

\section{INTRODUCTION}

Supernovae ( $\mathrm{SNe}$ ) play an important role in almost all areas of astrophysical research. Obviously central to stellar evolution, these explosions also trigger (and inhibit) star formation, produce heavy elements, dust and cosmic rays, and their energy input to the ISM is a crucial ingredient in galaxy formation. Supernovae are probably involved in the formation of neutron stars, black holes, and gamma-ray bursts (GRBs), and, as precision distance estimators, were instrumental in establishing the present cosmological standard model.

We recognize two physically defined classes: thermonuclear SNe (type Ia), occurring when a white dwarf (WD) star is pushed over the Chandrasekhar limit due to accretion from, or a merger with, a binary companion; and all other types of $\mathrm{SNe}$ (II, Ib, Ic) resulting from gravitational core-collapse of massive stars. The basic physical process of core-collapse SNe is known: a massive star (directly observed in a few nearby cases; Fig. 1) undergoes gravitational core-collapse forming a compact remnant. A mechanical 
shock, possibly assisted by neutrino energy deposition (e.g., the neutrino burst detected from SN 1987A), is launched, and ejects the envelope of the star.

However, despite this basic understanding, many fundamental questions remain. The continued failure of the spherical neutrino-driven shock mechanism to actually explode massive stars in 2- and 3-d numerical simulations, along with the advent of models invoking bipolar or jet-driven core-collapse induced explosions (Khokhlov et al. 1999; Burrows et al. 2006) has reopened the explosion mechanism question for core-collapse SNe. Different mechanisms predict unique mappings between progenitors and the resulting SNe (e.g., Heger et al. 2003), but we currently lack the corresponding observational basis to relate measured SN properties (brightness, light curve shape, spectroscopic abundances of $\mathrm{H}, \mathrm{He}$ and other elements) to those of the putative progenitor (age, initial mass, mass loss history, binarity), and thus constrain explosion models. The sum of our current knowledge is presented in Fig. 1. As can be seen there, a robust link between progenitor classes and $\mathrm{SN}$ types requires additional work. If the impact of the first progenitor-SN link (the blue supergiant progenitor of SN 1987A) is any indication, as the progenitor-SN map becomes more robust, it should provide a key input to SN models, and drive toward better understanding of SN physics. An additional benefit is that linking specific massive progenitor stars with different SN subtypes, in tandem with measurement of the rate and relative frequency and properties of subtypes (II-P, IIn, Ib, Ic, etc.) would ultimately allow to directly measure the stellar IMF out to high redshift (via detection of SNe, much brighter than any single star), and to calculate the role of core-collapse SNe in metal enrichment and energetics of the ISM, a prime topic of study with JWST, LSST, and the next generation of large telescopes.

With the goal of producing a robust progenitor-SN map (Fig. 1) to be used as a key to better understand core-collapse SN physics and the IMF, we are pursuing two parallel efforts. We are conducting a program to detect the progenitors of core-collapse $\mathrm{SNe}$ using a combination of pre-explosion HST images and post-explosion ground-based adaptive optics (AO) imaging (e.g., Gal-Yam et al. 2005; 2006). This program is aimed at providing additional links in the progenitor-SN map shown in Fig. 1. In parallel, we have been working on a large study of the typical properties and fractions of corecollapse $\mathrm{SNe}$ of various sub-types, in order to provide a well-defined subtype division (the currently used classification method of SNe is a mix of classification by spectra and by light-curve shapes) and a data base that can be used to interpret the results of large surveys at high redshift. Here we report the first results from this effort, the Caltech Core-Collapse Project (CCCP).

\section{THE CALTECH CORE-COLLAPSE PROJECT (CCCP)}

The CCCP (http: / / www . astro. caltech. edu/ avishay/cccp. html) is a large observational study of core-collapse SNe. Uniform, high-quality NIR and optical photometry and multi-epoch optical spectroscopy (Fig. 2) have been obtained using the 200" Hale and robotic 60" telescopes at Palomar, for a sample of 50 nearby core-collapse SNe (Table 1). The combination of both well-sampled optical light curves and multiepoch spectroscopy will allow to disentangle spectroscopically and photometrically based subtype definitions. Multi-epoch spectroscopy is crucial to identify transition 


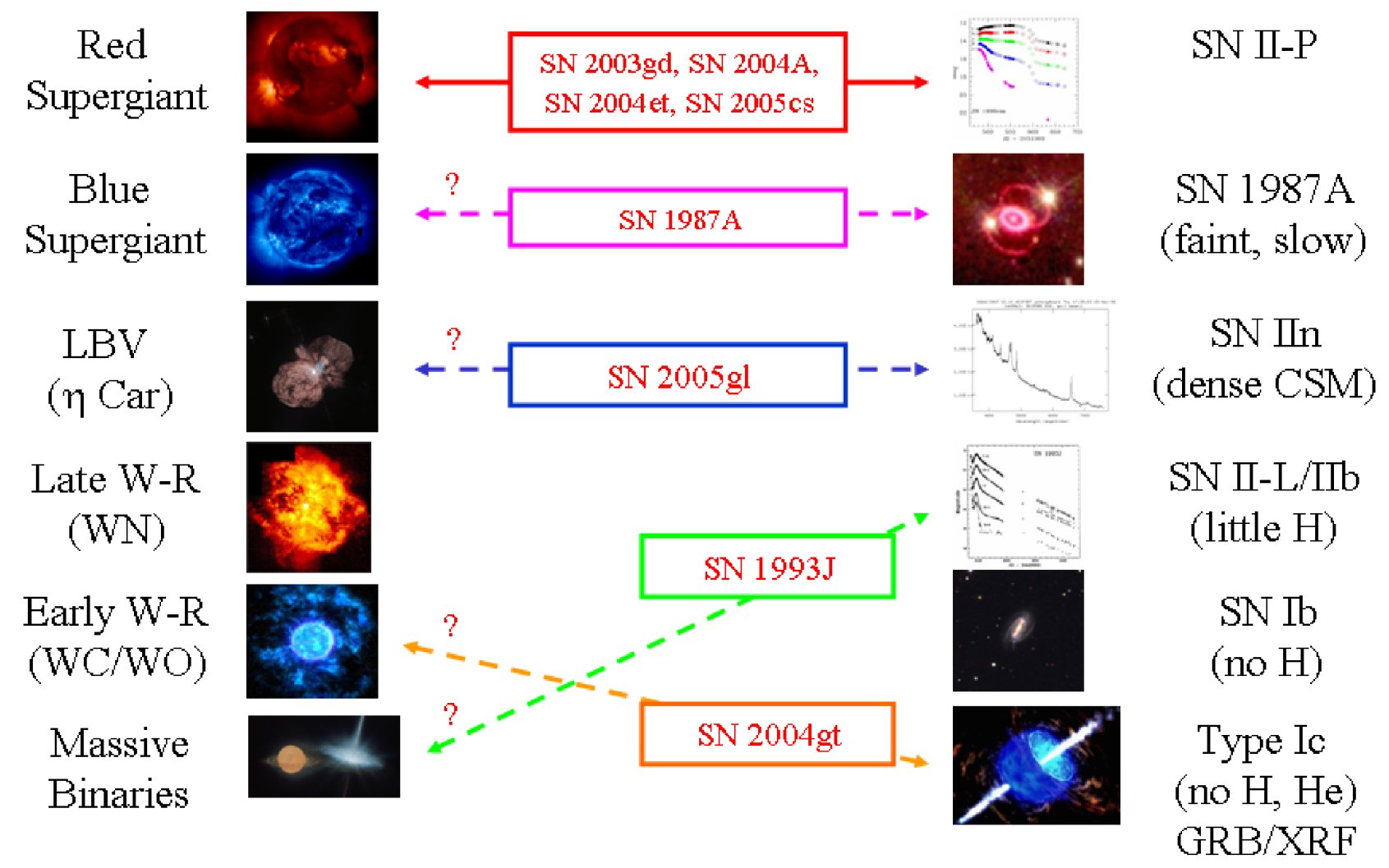

FIGURE 1. The progenitor-SN map based on the total census of progenitor observations (Gal-Yam et al. 2006 and references therein). The association of SNe II-P with red supergiants seems robust, all other associations are based on single events. Additional progenitor detections (of order 1-2 per year possible) are crucial for further progress. For example, conventional theory holds that only the most massive single stars give rise to $\mathrm{SNe} \mathrm{Ib} / \mathrm{c}$, but the theory is virtually unconstrained by observations - future progenitor identifications (or strict upper limits on the luminosity of these stars) should put our understanding of SNe to the test. The two most recent additions (SN 2004gt and SN 2005gl) are based on results from our AO program.

events that evolve among subtypes with time (e.g., SNe IIb, initially similar to H-rich $\mathrm{SNe}$ II, but later evolving into $\mathrm{H}$-poor and He-rich $\mathrm{SNe} \mathrm{Ib}$ - several such events have been identified among the CCCP sample). The CCCP SN sample includes every corecollapse SN discovered between July 2004 and September 2005 that was visible from Palomar, found shortly ( $<30$ days) after explosion (based on available pre-explosion photometry), and was closer than $\sim 120 \mathrm{Mpc}$. This complete sample allows, for the first time, a study of core-collapse $\mathrm{SNe}$ as a population, rather than as individual events. 

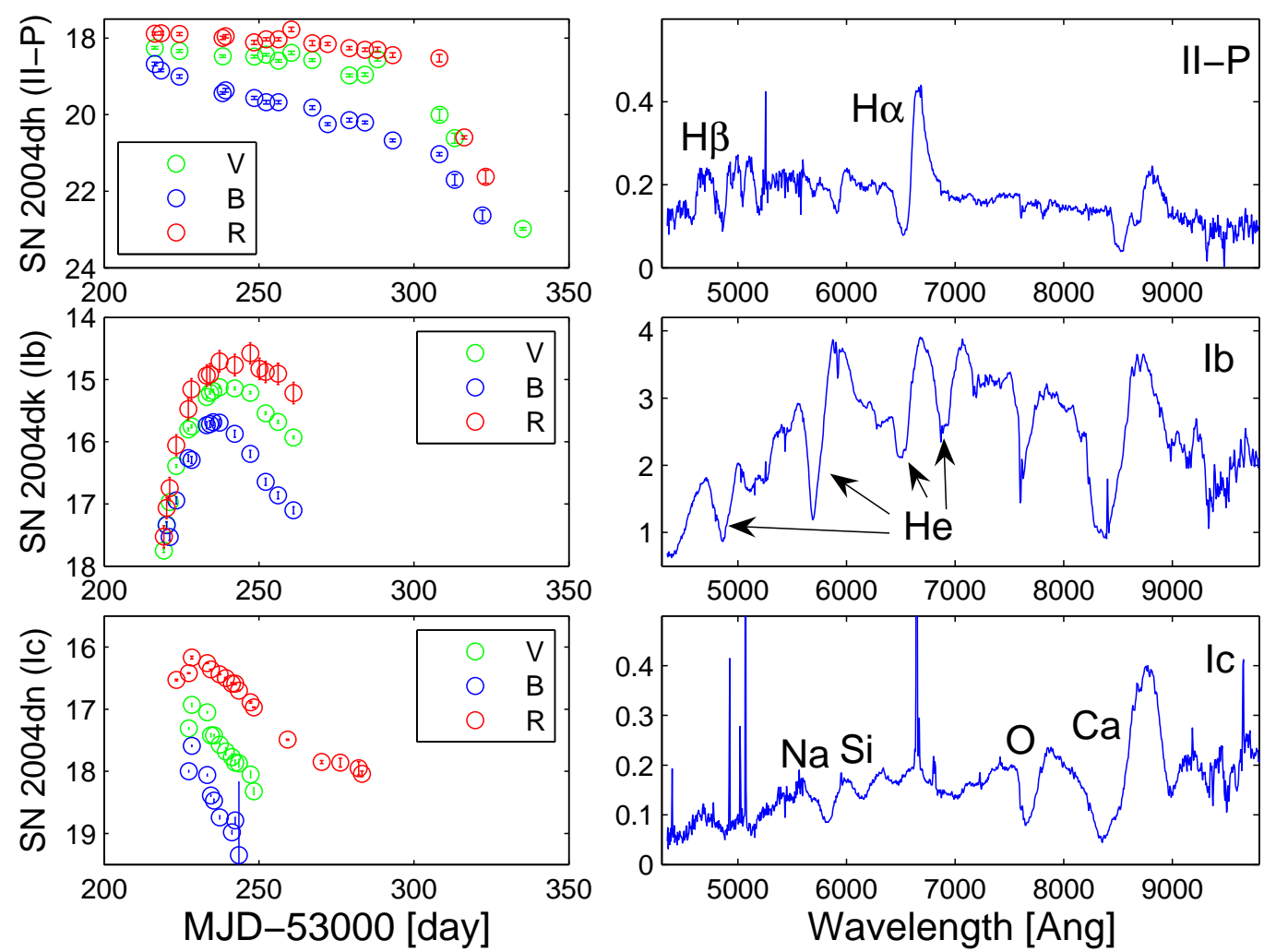

FIGURE 2. Example data for the first three CCCP SNe. Note well-sampled multi-color light curves and high S/N spectra, combined to accurately reveal the SN sub-type. Main species are marked. From Gal-Yam et al. 2007 (in preparation).

\section{PRELIMINARY RESULTS}

\section{The CCCP SN sample}

In Table 1 we list the full CCCP sample of SNe. Of the 50 core-collapse events, 37 are distributed among the various type II sub-classes, and 13 events are of types Ib and Ic. The sample includes two events (QuestSN1 and SNF0630) discovered using the Palomar 48" telescope by the Quest/SN factory consortium, and 48 events announced by various discovering groups in IAU circulars. Sample CCCP data (collected for the three first CCCP events) are shown in Fig. 2. Unfortunately, we were not able to secure such high-quality data for the entire CCCP sample, mainly due to the impact of the severe 2004-2005 California winter. However, data of similar quality exist for the majority of events studied. As can be seen from Fig. 2, our selection criteria for young events generally permitted us to obtain light curves that covered the epoch of peak brightness, and the large collecting area of the Palomar 200" enabled us to secure high S/N spectra.

As part of the activity associated with the CCCP effort we have also collected data for a number of events that did not satisfy our strict selection criteria. These include type II 
TABLE 1. The CCCP Supernova sample

\begin{tabular}{llllllllll}
\hline SN & Type & SN & Type & SN & Type & SN & Type & SN & Type \\
\hline 2004dh & II & $2004 \mathrm{dk}$ & Ib & $2004 \mathrm{dn}$ & Ic & $2004 \mathrm{dr}$ & II & $2004 \mathrm{du}$ & II \\
2004eb & II & $2004 \mathrm{em}$ & II & $2004 \mathrm{ek}$ & II & $2004 \mathrm{er}$ & II & $2004 \mathrm{et}$ & II \\
QuestSN1 & II & $2004 \mathrm{ex}$ & II & $2004 \mathrm{fc}$ & II & $2004 \mathrm{fe}$ & Ic & $2004 \mathrm{ff}$ & Ic \\
$2004 \mathrm{fx}$ & II & $2004 \mathrm{ge}$ & Ic & $2004 \mathrm{gq}$ & Ib/c & $2004 \mathrm{gt}$ & Ic & $2004 \mathrm{gv}$ & Ib/c \\
$2004 \mathrm{gy}$ & II & $2005 \mathrm{E}$ & Ib & $2005 \mathrm{H}$ & II & $2005 \mathrm{O}$ & Ib & $2005 \mathrm{U}$ & II \\
$2005 \mathrm{Y}$ & II & $2005 \mathrm{Z}$ & II & $2005 \mathrm{aa}$ & II & $2005 \mathrm{ab}$ & II & $2005 \mathrm{ad}$ & II \\
$2005 \mathrm{an}$ & II & $2005 \mathrm{ap}$ & II & $2005 \mathrm{ar}$ & Ib & $2005 \mathrm{au}$ & II & $2005 \mathrm{ay}$ & II \\
$2005 \mathrm{az}$ & Ib & $2005 \mathrm{ba}$ & II & $2005 \mathrm{bf}$ & Ib/c & $2005 \mathrm{bp}$ & II & $2005 \mathrm{bw}$ & II \\
$2005 \mathrm{bx}$ & II & $2005 \mathrm{by}$ & II & $2005 \mathrm{ci}$ & II & $2005 \mathrm{cl}$ & II & $2005 \mathrm{cp}$ & II \\
$2005 \mathrm{cs}$ & II & SNF0630 & II & $2005 \mathrm{db}$ & IIn & $2005 \mathrm{dp}$ & II & $2005 \mathrm{ds}$ & II \\
\hline II & 37 & Ib/c & 13 & Total & 50 & & & & \\
\hline
\end{tabular}

SNe 2004A (data published in Hendry et al. 2006), 2004T, 2004V and 2004Z observed as a pilot study of SNe II-P, observations of the bright nearby SN 2004dj (published in Leonard et al. 2006), observations of the peculiar type IIb SN 2004cs (published in Rajala et al. 2005), and observations of type IIn SN 2005gl for which we conducted a progenitor study (Gal-Yam et al. 2006).

\section{SN II Demography}

Analysis of the data collected by the CCCP program is in progress, and we are currently collecting the last observations required for field calibration and to serve as image-subtraction templates (only possible once the SNe have faded). A complete analysis of the data is therefore not yet possible. Here, we present a preliminary study of the first $\sim 1 / 3$ of our sample, for which light curves and reduced spectra are available, though not always fully calibrated. In particular, we have used the first 13 type II SNe for which good data exist to inspect the relative fractions of SNe II of the various subtypes. Table 2 presents our refined typing for these events. In the table we report both photometric type from our light curves $(\mathrm{P}=$ Plateau; $\mathrm{L}=$ rapidly declining; see below for an explanation about the events noted as peculiar) and the spectroscopic type (IIb = He lines appear with time; II-P = detailed analysis shows similarity to prototypical SNe II-P such as SN 1999em, references given; II - preliminary inspection does not show any noteworthy features).

As can be seen, of these first 13 events eight appear to be SNe II-P. Two events (SNe $2004 \mathrm{ek}$ and $2004 \mathrm{em}$ ) show peculiar light curves dominated by late-time humps rising on a timescale of tens of days (Fig. 3). SN 2004em appears to resemble a brighter version of SN 1987A, perhaps similar to SN 1998A (Pastorello et al. 2005), while SN 2004ek has an even more peculiar light curve. Regardless of their exact light curve shape, both events certainly do not decay rapidly as SNe II-L, and we can assume that sparser observations might have led to their classification as SNe II-P. We therefore conclude that $8(10)$ of our first 13 SNe II belong to the II-P subclass (depending on the inclusion of these two peculiar events), two events are of type Ib, and one (SN 2004dr) is of type 


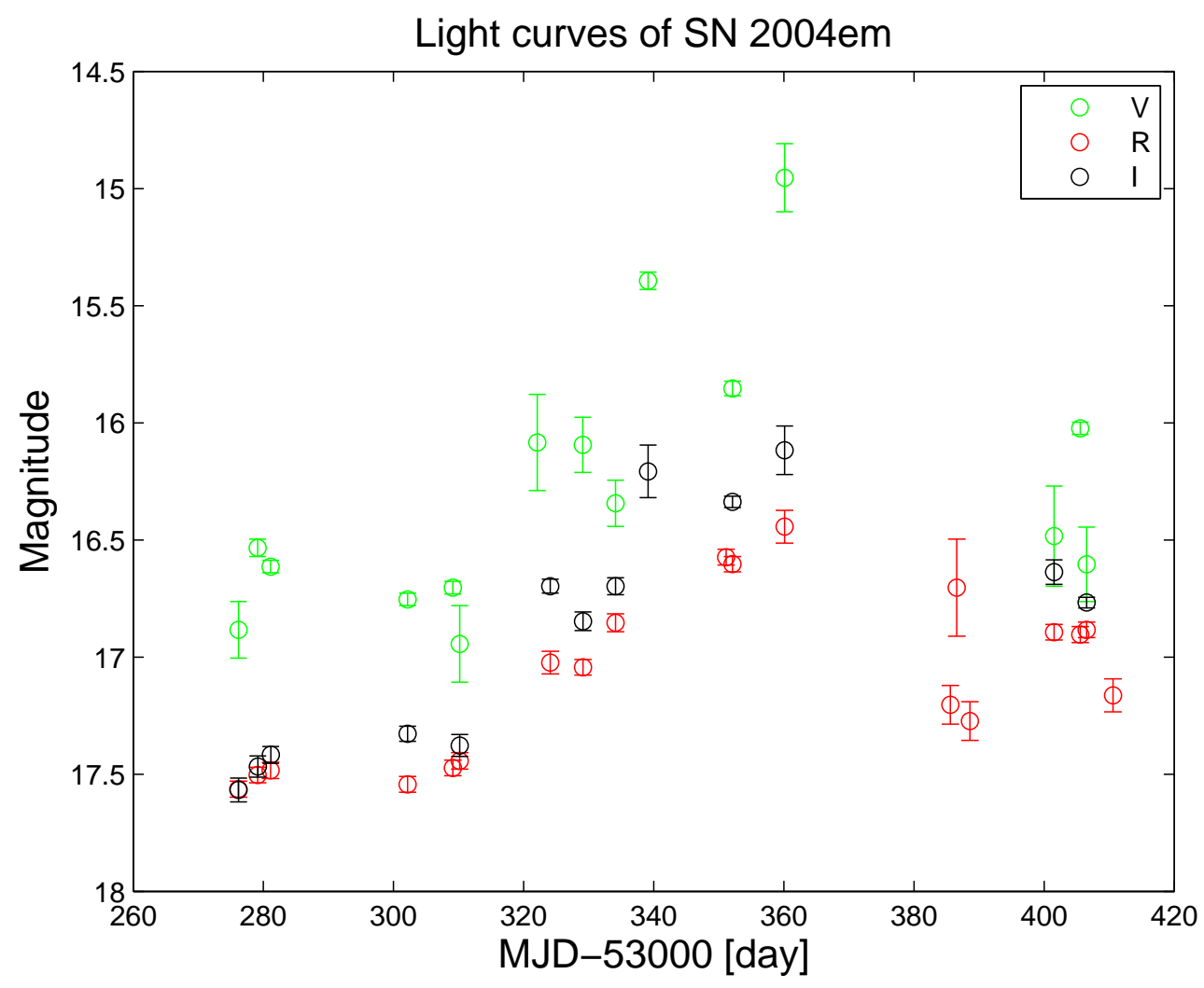

FIGURE 3. The peculiar light curve of SN $2004 \mathrm{em}$, based on preliminary CCCP photometry. Note the late-time hump peaking $\sim 3$ months after explosion.

II-L (which we here define as a SN with a rapidly declining light curve which does not show He signatures in its spectra). The more rare SNe IIn are not represented in this first sub-sample of SNe from the CCCP (though several such events are included in the full sample; Table 1). Assuming that our sample does not suffer from strong selection effect (being almost volume, rather than flux, limited) we tentatively deduce that a fraction $f=60-75 \%$ of SNe II, and $\sim 50 \%$ of core-collapse SNe, are of type II-P.

Interpreting this result in the context of the progenitor-SN map (Fig. 1), which links SNe II-P with red supergiants with an initial low main sequence mass $\left(8-15 \mathrm{M}_{\odot}\right)$, we calculate the fraction of such stars from the total population of core-collapse-producing stars $\left(\mathrm{M}>8 \mathrm{M}_{\odot}\right)$. Assuming a Salpeter mass function, we find the the expected fraction of SNe II-P (assuming all SNe II-P come from low-mass red supergiants, and that all stars in the above mass range explode as SNe II-P) is $f=57 \%$. The nice agreement with our preliminary findings above is encouraging, and may tell us that the above assumptions are correct. In particular, if most $8-15 \mathrm{M}_{\odot}$ stars explode as SNe II-P, perhaps stars in this mass range, even if in binary systems, do not contribute significantly to the population of stripped $(\mathrm{Ib} / \mathrm{c}) \mathrm{SNe}$. 
TABLE 2. Type II Supernovae from the CCCP

\begin{tabular}{llll}
\hline SN & Photo Type & Spec Type & Reference \\
\hline $2004 \mathrm{~A}$ & P & II-P & Hendry et al. 2006 \\
$2004 \mathrm{cs}$ & L & IIb & Rajala et al. 2005 \\
$2004 \mathrm{dh}$ & $\mathrm{P}$ & II-P & Nugent et al. 2006 \\
$2004 \mathrm{dr}$ & L & II & \\
$2004 \mathrm{du}$ & $\mathrm{P}$ & II & \\
$2004 \mathrm{ek}$ & $\mathrm{P}(\mathrm{pec})$ & II & \\
$2004 \mathrm{em}$ & $\mathrm{P}(\mathrm{pec})$ & II & \\
$2004 \mathrm{er}$ & $\mathrm{P}$ & II & \\
$2004 \mathrm{et}$ & $\mathrm{P}$ & II-P & Li et al. 2005 \\
$2004 \mathrm{ex}$ & L & IIb & \\
$2004 \mathrm{fc}$ & $\mathrm{P}$ & II & \\
$2004 \mathrm{fx}$ & $\mathrm{P}$ & II-P & Hamuy et al. 2006 \\
$2005 \mathrm{ay}$ & $\mathrm{P}$ & II-P & Bufano et al. 2006 \\
\hline
\end{tabular}

\section{CONCLUSIONS}

We have conducted a large observational program focused on core-collapse SNe, the CCCP. $50 \mathrm{SNe}$ have been observed, and data analysis is in progress. While exploiting the full potential of this large data set will require additional time and effort, preliminary results from the CCCP and associated projects are emerging. Here, we have shown an initial inspection of type II SN demographics, which appear to be consistent with the idea that SNe II-P are associated with low-mass progenitor stars.

\section{ACKNOWLEDGMENTS}

A.G. acknowledges support by NASA through Hubble Fellowship grant \#HST-HF01158.01-A awarded by STScI, which is operated by AURA, Inc., for NASA, under contract NAS 5-26555. A.G. further acknowledges the hospitality of the community of Cefalu and the efforts of the organizers of the 2006 Cefalu international astronomy conference, during which this work has come to fruition. D.C.L. acknowledges support from a National Science Foundation (NSF) Astronomy and Astrophysics Postdoctoral Fellowship (award AST-0401479), under which part of this work was completed. D.J.S. acknowledges support provided by NASA through Chandra Postdoctoral Fellowship grant number PF5-60041.

\section{REFERENCES}

1. Bufano, F. M., Turatto, M., Zampieri, L., \& Gal-Yam, A. 2006, Supernovae: One Millennium After SN1006, 26th meeting of the IAU, Joint Discussion 9, 17-18 August 2006, Prague, Czech Republic, JD09, \#36, 9,

2. Burrows, A., Livne, E., Dessart, L., Ott, C. D., \& Murphy, J. 2006, ApJ, 640, 878

3. Gal-Yam, A., et al. 2005, ApJL, 630, L29

4. Gal-Yam, A. et al. 2006, apJ, in press, astro-ph/0608029 
5. Hamuy, M., et al. 2006, PASP, 118, 2

6. Heger, A., Fryer, C. L., Woosley, S. E., Langer, N., \& Hartmann, D. H. 2003, ApJ, 591, 288

7. Hendry, M. A., et al. 2006, MNRAS, 369, 1303

8. Khokhlov, A. M., Höflich, P. A., Oran, E. S., Wheeler, J. C., Wang, L., \& Chtchelkanova, A. Y. 1999, ApJL, 524, L107

9. Leonard, D. C., et al. 2006, Nature, 440, 505

10. Li, W., Van Dyk, S. D., Filippenko, A. V., \& Cuillandre, J.-C. 2005, PASP, 117, 121

11. Nugent, P., et al. 2006, ApJ, 645, 841

12. Pastorello, A., et al. 2005, MNRAS, 360, 950

13. Rajala, A. M. et al. 2005, PASP, 117, 132 